\title{
edoc
}

Institutional Repository of the University of Basel

University Library

Schoenbeinstrasse 18-20

CH-4056 Basel, Switzerland

http://edoc.unibas.ch/

\section{Konstellationen und Grenzen des Tragischen. Figuren der Negativität}

\author{
Angehrn, Emil
}

Posted at edoc, University of Basel

Official URL: http://edoc.unibas.ch/dok/A5848406

Originally published as:

Angehrn, Emil. (2011) Konstellationen und Grenzen des Tragischen : Figuren der Negativität. In: Die Philosophie des Tragischen : Schopenhauer - Schelling - Nietzsche. Berlin, S. 39-59.

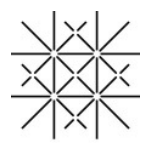




\title{
Konstellationen und Grenzen des Tragischen. Figuren der Negativität
}

\author{
Emil Angehrn
}

Das Scheitern ist den Menschen vertrauter als das Gelingen, das Leiden näher als das Glück. Auch die aktuelle Konjunktur der Beschäftigung mit Glück und Lebenskunst erscheint eher wie eine temporäre Gegenbewegung in der Geschichte der Besinnung auf den Menschen. Die Befindlichkeiten, in denen der Mensch seines Seins gewahr wird, sind nach der Existenzphilosophie vorzugsweise Negativaffekte wie Angst, Verzweiflung, Langeweile. Unter vielfältigsten Abschattungen wird die Negativität menschlicher Existenz erfahren und beschrieben. Vergänglichkeit, Haltlosigkeit, Entfremdung sind Facetten solcher Beschreibung. Es sind Indizien eines fundamentalen Ungenügens, eines Verfehlens, das konstitutiv zur menschlichen Existenz gehört.

Vielfach ist solches Verfehlen im Zeichen des Tragischen gedeutet worden. Maurice Maeterlinck hat von einer "Tragik des Alltags“ gesprochen, die mit dem Leben als solchem verflochten ist und von der er meint, dass sie ,viel wahrer und tiefer ist und unserem wahren Wesen weit mehr entspricht als die Tragik der großen Abenteuer", der ewigen Konflikte und überwältigenden Schmerzen. ${ }^{1}$ Allerdings scheint klar, dass in einem engeren Verständnis nicht all diesem die Aura des Tragischen zukommt. Nicht alles, worunter wir leiden oder was wir als schicksalhafte Versagung erleben, ist als tragisch zu bezeichnen.

Die Frage, der ich im Folgenden nachgehen möchte, gilt den spezifischen Merkmalen des Tragischen und genauer dem Verhältnis von Tragik und Negativität. Sie fragt danach, was die besondere Negativität des Tragischen auszeichnet und wie dessen Ort im menschlichen Dasein bestimmt ist. Sie fragt auch danach, welches die Grenzen des Tragischen sind. Zwischen existentieller Negativität und Tragik besteht nicht einfach Deckungsgleichheit, sondern gleichsam eine zweifache Inkongruenz.

1 Maurice Maeterlinck: Le Tragique quotidien. In: ders.: Le Trésor des humbles. Essai (1896). Brüssel 1986, Neuaufl. 1998, S. 99-110, hier S. 101 [Übers. v. Verf.]. 
Nicht jede Erfahrung des Schmerzes, des Verlusts und Vergehens ist tragisches Bewusstsein. Umgekehrt ist das Tragische nicht notwendig ein Letztes. In der Moderne wird das tragische Bewusstsein teils überwunden oder aufgelöst; teils kommt ein Negatives jenseits des Tragischen in den Blick. In Frage steht nicht eine bloße Begriffsklärung; die Verdeutlichung des Verhältnisses von Tragik und Negativität gilt zugleich dem Bemühen, sich im Ausgang vom Tragischen über Bedingungen menschlichen Selbstseins und dessen konstitutive Negativität zu verständigen.

Es liegt auf der Hand, dass jede differenzierende Verhältnisbestimmung von Tragik und Negativität Schematisierungen in Anspruch nimmt, die nicht zuletzt einen historischen Index haben. Die Geschichte der Tragödie - etwa in der modernen Transformation der antiken Tragödie - ist selbst ein Prozess, in dem Aspekte auseinandergehalten und neu konstelliert werden, die sich je nachdem im Binnenraum des Tragischen situieren oder als dessen Anderes auftreten. Die angedeutete Nichtkongruenz lässt sich zum Teil auch als eine zwischen Versionen des Tragischen oder der Tragödie lesen. Jede Diskussion geht explizit oder implizit von idealtypischen Modellen aus, sei es, dass sie sich an der griechischen Tragödie selbst (von der uns ohnehin nur ein geringer Teil überliefert ist), an herausgehobenen klassischen Werken (etwa König Ödipus oder Antigone), an der Geschichte der Tragödie im Ganzen oder an klassischen Theorien der Tragödie (etwa von Aristoteles oder Hegel), die ihrerseits selektiv angelegt sind, ${ }^{2}$ orientiert. Für das Folgende ist von einem Paradigma auszugehen, das im Wesentlichen durch die klassische griechische Dichtung gegeben ist, wobei als exemplarische Bezugspunkte die Dramen von Aischylos und Sophokles dienen. Mit Bezug auf sie ist zu fragen, welches die charakteristischen Merkmale des Tragischen sind.

$\mathrm{Zu}$ diesen zählen zuerst Merkmale, welche die conditio humana auszeichnen, sozusagen die tragische Verfassung des menschlichen Seins ausmachen (1.). Zugleich thematisiert die Tragödie, in welcher Weise Subjekte sich zu dieser Verfassung verhalten, wie sie sich handelnd, erkennend und deutend mit der Tragik der Existenz auseinandersetzen und in dieser Auseinandersetzung ihr Selbstsein gewinnen (2.). Im Blick auf diese Gesamtkonstellation ist nach den Grenzen des Tragischen (3.) und dem Verhältnis von Negativismus und Tragik (4.) zu fragen.

2 Für die Festlegung durch Aristoteles (z. B. im Vergleich mit Gorgias) vgl. in diesem Band: Bernhard Zimmermann: Über das Tragische bei den Griechen. 


\section{Die tragische Verfassung der Existenz}

Die Verfassung des menschlichen Seins kommt in der Tragödie unter vielfältigen Aspekten zum Ausdruck, die sich um drei Gravitationszentren gruppieren lassen. Die Tragik der Existenz ist durch die Endlichkeit alles Menschlichen, die innere Konfliktualität des Handelns und die unhintergehbare Schuldhaftigkeit des Daseins bedingt.

\subsection{Endlichkeit, Ohnmacht, Leiden}

Die Urerfahrung, die sich schon im Mythos Ausdruck verschafft und in der Tragödie ihren Widerhall findet, ist die der Nichtigkeit alles Menschlichen. Sie ist sowohl die Erfahrung der Ohnmacht gegenüber der Willkür der Götter und der Herrschaft des Schicksals wie das Erleben der eigenen, inneren Hinfälligkeit des menschlichen Seins. Dass die Menschen sterblich sind, ist ihr basalstes Leiden und ihr erstes Differenzmerkmal gegenüber den Göttern. Die Herrschaft der Zeit ist die erste Fremdherrschaft über den Menschen: ,allein die Götter sind vom Alter allezeit verschont und auch vom Tod. Das übrige zerstört die Allgewalt der Zeit." ${ }^{\text {3 }}$ Die Sterblichkeit ist die elementarste Hinfälligkeit des Menschen, tiefer noch als die Zerbrechlichkeit von Glück und Ansehen, die das Elend des Menschen bedingt. Allem zugrunde liegt der Antagonismus von Sein und Nichtsein, der in den Gegensätzen von Bestehen und Vergehen, Festigkeit und Wandel wiederkehrt (wie Antigone den menschlichen Satzungen die „ewige[n], göttliche[n] Gesetze“ gegenüberstellt, die ,immerdar bestehn“). ${ }^{4}$ Die Urangst vor dem Nichts, die sich ebenso im Schrecken vor dem Chaos, dem Gestalt- und Grenzenlosen äußert, die Erfahrung der Haltlosigkeit und Leere sind Erlebensformen dieser Bedrohung, die nicht einem bestimmten Ziel oder $\mathrm{Zu}-$ stand, sondern dem Sein des Subjekts als solchem gilt.

Nietzsche hat dieses Bedrohtsein in entgegengesetzten Gestalten in die Tiefenschicht des Tragischen eingezeichnet. Die Dynamik des Apollinischen und Dionysischen verknüpft gegenläufige Utopien - die

3 Sophokles, Oidipus auf Kolonos, V. 607-609 (Sophokles' Werke werden zitiert nach: Sophokles: Dramen. Griech. u. dt. Hg. u. übers. v. Wilhelm Willige, überarb. v. Karl Bayer. Mit Anm. u. e. Nachw. v. Bernhard Zimmermann. München/Zürich ${ }^{2}$ 1985).

4 Sophokles, Antigone, V. 454-456. 
Sehnsucht des Aufgehens im Ganzen und das Streben nach der individuierenden Gestalt -, denen gleichzeitig Formen des Selbstverlusts und des Schreckens innewohnen: das „ungeheure Grausen" angesichts des Verlusts von Form und Bestimmtheit auf der einen Seite, die trennende Individuation als „Quell und Urgrund alles Leidens“ auf der anderen. ${ }^{5}$ Das Einssein mit dem Ganzen und die Befreiung aus ihm werden in abgründigem Zwiespalt sowohl als Erlösung wie als Vernichtung erlebt. Im Negativen sind es entgegengesetzte Formen des Selbstverlusts, die in ihrer Gegenläufigkeit eine Art Fatalität des unausweichlichen Untergangs verkörpern. Das Individuum ist sowohl durch die Vereinigung wie die Trennung in seinem Sein bedroht.

Nach einer dritten Hinsicht ist der Mensch nicht nur in seiner Existenz und in seiner Individuation, sondern in seinem subjektiven Selbstsein dem Scheitern ausgesetzt. Als Subjekt, das in seinem Selbstverhältnis mit sich eins werden will, droht ihm ein zweifaches Sichverfehlen. Das Subjekt will einerseits sein eigener Ursprung sein, sich selbst bestimmen und entwerfen, andererseits in seinem Tun mit sich zur Deckung gelangen und seine Ziele verwirklichen. Dem Streben nach Ursprünglichkeit entspricht auf der Gegenseite das Verlangen nach Erfüllung und Ganzheit. Doch nach beiden Seiten entgleitet ihm die Selbstkoinzidenz. Uneinholbar bleibt die retrospektive wie die prospektive Selbstpräsenz, die Identität des Anfangs wie des Abschlusses: Der Mensch kommt nie ganz aus sich und gelangt nie ganz zu sich. Auch diese ,Schwäche' des Selbst, die in neueren Theorien reflektiert wird, steht für ein ursprüngliches Defizit und ein originäres Leiden, mit dem das Selbst in seinem Tun und Wollen konfrontiert ist.

All dies sind Formen des Mangels, die mit dem menschlichen Sein als solchem gegeben sind. Es sind Erfahrungen des Leidens, des Ausgeliefertseins und der Haltlosigkeit, des unstillbaren Begehrens und der Nichtganzheit der Existenz. All diese Aspekte werden in der alten Literatur - teils im Mythos, teils in der archaischen Lyrik, teils in der Tragödie - thematisch und zum Kern der Reflexion über den Menschen. Im Ganzen repräsentieren sie eine seinsmäßige Negativität, die sich dem Ideal wahrhaften Seins und erfüllten Lebens widersetzt. Für sich genommen, sind es noch nicht spezifische Merkmale des Tragischen, sofern ihnen der Zug der inneren Widersprüchlichkeit, der das Tragische kennzeichnet, nicht anhaftet. Doch kommen sie innerhalb der Tragödie mit zur Sprache, gehören sie mit zur Negativität der tragischen Kon-

5 Nietzsche, GT 1, KSA 1, S. 28 u. GT 2, KSA 1, S. 72. 
stellation. Die Hinfälligkeit und konstitutive Mangelhaftigkeit der Existenz bildet den Rahmen und gleichsam den seinsmäßigen Grund jener spezifischen Ausweglosigkeit, in welche sich das tragische Handeln verstrickt. In einem schwächeren Sinn können wir zwar auch in diesen Erfahrungen eine Art Gegenläufigkeit des Strebens, ein konstitutives Sich-nicht-Erfüllen-Können ausmachen, das mit der strukturellen Verfassung des Tragischen verwandt scheint. Doch ist es eine Unstimmigkeit, die in der Endlichkeit als solcher liegt und noch nicht jene Härte und Unversöhntheit besitzt, die das Tragische in seinem Kern bestimmt.

\subsection{Konfliktualität des Handelns}

Über solche Defizienz gehen jene Formen der Nichtidentität hinaus, die nicht der Ohnmacht, sondern der inneren Konfliktualität und Zerrissenheit des menschlichen Seins geschuldet sind. Ein Kern des Tragischen liegt für die klassische Tragödientheorie in der Kollision der Werte und Pflichten, die sittlichem Handeln innewohnt. Nicht der Kampf feindlicher Mächte oder der Antagonismus zwischen Gut und Böse, sondern die unausweichliche Kollision positiver Werte, die ob ihrer Einseitigkeit nicht nebeneinander bestehen können, macht das Tragische des Konflikts aus. Tragisch ist das Handeln, das in der Verfolgung eines Zwecks notwendig einen andern Wert oder berechtigten Anspruch verletzt. Para"digmatisch sind Konstellationen, in denen nicht einfach die Unvereinbarkeit partikularer Wertsetzungen, sondern gleichsam notwendiger Gegensätze, die in ihrem Spannungsverhältnis ein Ganzes umreißen, ausgetragen wird - so etwa der Gegensatz zwischen der natürlichen Pietät der Familie und der Allgemeinheit des Staats in Sophokles' Antigone. Generell ist der mythische Polytheismus Sinnbild dieser „Grundunstimmigkeit der Welt" ${ }^{6}$ welcher der Mensch ebenso ausgeliefert ist wie der Übermacht des Schicksals und die Max Weber analog in ,,unlöslichem Kampf" der pluralen Wertordnungen wahrnimmt. ${ }^{7}$ Die Unvollendetheit und Partialität ist nicht einfach eine strukturelle, sondern eine normative, eine Selbstverunmöglichung des Guten, die auf das Subjekt zurückschlägt und seine ethische Selbstverwirklichung untergräbt. Der Mensch kann

6 Karl Jaspers: Über das Tragische. München 1952, S. 13.

7 Max Weber: Wissenschaft als Beruf. In: ders.: Gesammelte Aufsätze zur Wissenschaftslehre. Hg. v. Johannes Winckelmann. Tübingen ${ }^{4} 1973$, S. 582-613, hier S. 603. 
nicht einfach in der einseitigen Realisierung des Sittlichen aufgehen und darin seine Erfüllung finden. Dieser Verwirklichung, auch wenn sie einen Idealtypus heroischer Existenz zeichnet, haftet die Unversöhntheit des Ganzen als eigener Makel an, der das Subjekt am Ende dem Untergang weiht - wie nicht nur Antigone das Unheil ereilt, sondern auch Kreon, der sich in unlösbare Schuld verstrickt und zuletzt ganz vernichtet ist, „nicht mehr als ein Nichts. “8

Unter verschiedenen Aspekten wird das Tragische als Einheitsverlust erfahren. War es zunächst die Erfahrung der Unerfulltheit, so geht es nun um eine Inkonsistenz und einen inneren Zwiespalt, in verschärfter Form um ein in sich widersprüchliches Wollen. Darin äußert sich eine Aporetik, die in analogen Figuren auch unabhängig von der Tragik in Konzepten subjektiven Selbstseins im Nebeneinander unvereinbarer Selbstbeschreibungen herausgestellt wird. ${ }^{9}$ Im Fall der tragischen Konfliktualität geht es um die Verstrickung in einen Selbstwiderspruch, worin das Leiden als Resultat des eigenen Tuns und Wollens erscheint. Tragisch ist nicht, dass die Durchsetzung unserer Vorhaben immer unzulänglich bleibt, sondern dass das Wollen sich in sich selbst verkehrt und das Tun zur Zerstörung wird. Die tragische Konfliktualität steht für einen Selbstwiderspruch, der in Zerrissenheit und Selbstentfremdung resultiert.

\subsection{Schuldhaftigkeit des Daseins}

Einen Schritt weiter geht die Exploration des Tragischen dort, wo die Negativität nicht nur als faktisch erlittene und selbst bewirkte, sondern als Folge von Schuld und Unrecht erfahren wird. Dabei tritt die Qualität des Schuldhaften in variierenden Abschattungen in Erscheinung. In basalster Weise wird das Leiden als Folge, teils als Sühne eines Vergehens, teils aber auch als Folge eines bloßen Schuldspruchs, als grundlos erlittenes Unrecht erfahren. Für Nietzsche ist das ursprüngliche Leiden als Folge der Individuation die Kehrseite eines ursprünglichsten Frevels, ist der unausweichliche Untergang die Buße für die Ursünde eines Abfallens vom

8 Sophokles, Antigone, V. 1325.

9 Dieter Henrich verweist auf gegenläufige Orientierungen und theoretische Ausgriffe, in denen das Subjekt seine Stellung im Ganzen reflektiert und die es nicht zur durchsichtigen Einheit zusammenzubringen vermag: Vgl. Dieter Henrich: Denken und Selbstsein. Vorlesungen über Subjektivität. Frankfurt a.M. 2007, S. 44 f. u. S. 49 f. 
Ganzen. ${ }^{10}$ In Schöpfungsmythen wird die Sterblichkeit zuweilen als grausam über die Menschen verhängtes Los, als Unrecht der von den Göttern vorgenommenen Teilung angeprangert. In grundlegender Weise begegnet uns in der Tragödie die Unausweichlichkeit des Schuldigwerdens als Moment des Menschseins. Das Tragische steht im Zeichen der Schuld, es kennzeichnet den Stand der Unerlöstheit. ${ }^{11}$ Das Leiden des tragischen Helden, das nach Aristoteles Furcht und Jammer erregt, ist nicht einfach ein ihm von außen zugefügtes Unheil, sondern das Büßen für ein Vergehen, das Abtragen einer Schuld: ${ }^{12}$ doch einer Schuld, die wie ein Verhängnis am Handelnden haftet, sei es, dass sie einem göttlichen Fluch entstammt, sei es, dass er durch seine Herkunft in sie verstrickt ist oder dass er durch sein eigenes Handeln, wie Ödipus, unwissentlich-unfreiwillig Schuld auf sich geladen hat - die aber nach Sühne verlangt und für die er einzustehen hat. Nicht einfach, dass er für die Fehler anderer einzustehen, ohne moralisches Verschulden ${ }^{13}$ die Strafe für fremdes Vergehen zu tragen hat, sondern dass er die Schuld und das Vergehen selbst auf sich nehmen muss, macht die wirkliche Härte des tragischen Schicksals aus. Zugespitzt formuliert: Nicht das schuldlose Leiden, sondern die schuldlose Schuld bildet den Kern jener Aporetik, die sich im Tragischen offenbart. Sie ist ein Stein des Anstoßes nicht nur für ein modernes Moralverständnis, sondern für das tragische Bewusstsein selbst.

Von der Unzulänglichkeit des eigenen Seins und Könnens gelangen wir hier zu einer abgründigeren, inneren Defizienz des Subjekts, die ein

10 So Nietzsches (strittige) Lesart des Anaximander-Fragments : Nietzsche, PHG 3 , KSA 1, S. $817 \mathrm{f}$.

11 Walter Benjamin bezeichnet das Verhältnis der Sprachen nach dem Sündenfall und der Sprachenzerstreuung nach Babel als ,tragische[s] Verhältnis der Sprachen" (Walter Benjamin: Über Sprache überhaupt und über die Sprache der Menschen. In: ders.: Gesammelte Schriften. Hg. v. Rolf Tiedemann u.a. Frankfurt a.M. 1972 ff., Bd. II.1, S. 140-157, hier S. 156).

12 Anders Christoph Menke, der in der Vorstellung einer Schuld des tragischen Helden ein Missverständnis der aristotelischen Rede von einem Fehler (hamartia) sieht (vgl. Christoph Menke: Die Gegenwart der Tragödie. Versuch über Urteil und Spiel. Frankfurt a.M. 2005, S. 80). Klar ist allerdings, dass es nicht um ein ,technisches“, sondern ein normatives Verfehlen geht, so dass auch der „Umschlag ins Unglück" nicht eine bloß äußere Handlungsfolge oder Fatalität ist.

13 Der tragische Held erleidet nach Aristoteles (Aristoteles, Poetik, Kap. 13, 1453a9) „nicht wegen seiner Schlechtigkeit und Gemeinheit einen Umschlag ins Unglïck" (Aristoteles' Poetik wird zitiert nach: Aristoteles: Poetik. Griech. u. dt. Übers. u. hg.v. Manfred Fuhrmann. Stuttgart 1982). 
spezifisches Merkmal des Tragischen ausmacht. Ihre Abgründigkeit ist jener verwandt, die in der Metaphysik den Ursprung und die Intelligibilität des Bösen kennzeichnet. In letzter Zuspitzung kommt das $\mathrm{Ne}-$ gative in der Verflechtung zwischen dem Leiden und dem Bösen in den Blick. Für das normale Empfinden wie den Anspruch der Vernunft ist das Leiden Unschuldiger das Unerträgliche. Hiob ist eine Herausforderung für die Religion wie das Erdbeben von Lissabon für die Aufklärung und das Leiden der Kinder unter der Pest für die existentialistische Sinnfrage. Vertieft wird das Leiden durch die Übernahme der nicht-verschuldeten Schuld, durch welche das Subjekt gewissermaßen zum Urheber des eigenen Leidens wird - eine Negativität, die ihrerseits gesteigert wird, wenn solche Schuld dem Schuldspruch einer missgünstigen Gottheit entstammt. Es ist dies nach Ricœur die abgründigste Erfahrung der Sinnlosigkeit, die nicht nur im Fehlen von Sinn, sondern in dessen Verunmöglichung durch die Negativität des Grundes, letztlich in der Urangst vor einem bösen Urgrund liegt. In der Angst, ob nicht der Gott böse sei, sieht Ricœur die tiefste Verunsicherung, der der Mensch ausgesetzt sein kann. ${ }^{14}$

Auch wenn die Konfrontation mit dem Bösen und dem Übel in der Tragödie und im Mythos nicht in gleicher Weise wie in der spekulativen Philosophie und der Theodizee begrifflich entfaltet wird, ist sie im Medium von Erzählung, dramatischer Handlung und Symbol in prägnanter Weise gegenwärtig. ${ }^{15}$ Die Erfahrung des Negativen wird darin in seiner Tiefe aufgenommen und in das Verständnis des Menschen und der Welt integriert. Das tragische Bewusstsein ist eines, das die Unhintergehbarkeit von Ohnmacht, Konfliktualität, Schuld und Leiden auf sich nimmt und als Kern und Grund des menschlichen Seins anerkennt.

14 Vgl. Paul Ricour: Vraie et fausse angoisse (1953). In: ders. : Histoire et vérité. Paris ${ }^{3} 1967$, S. 357-377, hier S. 373-375.

15 Vgl. Paul Ricœur: Philosophie de la volonté. Tome II. Finitude et culpabilité. - I. L'homme faillible; II. La symbolique du mal. Paris 1960. Vgl. Emil Angehrn: Compréhension de soi et altérité. Entre phénoménologie et herméneutique. In: Raphaël Célis/Muriel Gilbert (Hg.): Paul Ricœur interprète de la tradition occidentale. Erscheint Paris 2011. 


\section{Die Auseinandersetzung mit dem Tragischen}

Zur Charakterisierung der Tragik gehört die Auseinandersetzung mit ihr: $\mathrm{Zu}$ präzisieren ist, wie sich der Mensch erkennend, deutend und handelnd zur tragischen Verfassung der conditio humana verhält. Dieses Verhalten wird in der Tragödie als Teil des tragischen Erlebens und Handelns mit gegenwärtig.

\subsection{Auflehnung und Selbstbehauptung}

Eine erste Reaktion ist die Auflehnung und Selbstbehauptung des Menschen gegen das über ihn verhängte Los. Schon die Schilderung der Unerbittlichkeit des Schicksals, des grundlosen Leidens und schuldlosen Schuldigseins ist ein Einspruch gegen die mythische Rechtsordnung. Die Tragödie selbst ist nicht nur Darstellung von Ausweglosigkeit, Schuld und Leiden, sie ist ebenso Ausdruck des Unrechts, das dem Helden geschieht, der Härte, Anstößigkeit der Ordnung, der er ausgeliefert ist. Vielfach bringt sie die direkte Auflehnung zum Ausdruck: die Klage, der Protest, ja, die Schmähung der Götter (etwa bei Prometheus) sind Reaktionen auf erlittenes, nicht-gerechtfertigtes Leid. Nicht selten gehen sie mit Charaktereigenschaften wie Eigensinn, Eigenwilligkeit, Unnachgiebigkeit einher, teils negativen Abschattungen der Charakterstärke und Selbstbehauptung, die zur Selbstwerdung des Subjekts gehört.

\subsection{Integration des Negativen und Versöhnung}

Kehrseite der Auflehnung ist das Aufsichnehmen des Negativen. Die Endlichkeit, die Konfliktualität, die Sterblichkeit auszuhalten und ins eigene Sein zu integrieren macht die tragische Größe aus und bildet eine Forderung an das menschliche Leben. Emphatisch bringt sie Hegel zur Sprache:

Nicht das Leben, das sich vor dem Tode scheut und von der Verwüstung rein bewahrt, sondern das ihn erträgt und in ihm sich erhält, ist das Leben des Geistes. Er gewinnt seine Wahrheit nur, indem er in der absoluten Zerrissenheit sich selbst findet. ${ }^{16}$

16 Hegel, Phän., TWA 3, S. 36. 
Die Kraft, mit der ,ungeheure[n] Macht des Negativen“ zurechtzukommen, wohnt nach Hegel nur jenem Leben inne, das , dem Negativen ins Angesicht schaut, bei ihm verweilt. ${ }^{\text {"17 }}$ Doch ist das Ziel nicht nur eine Bewältigung und ein Bezwingen, ein Aushaltenkönnen ohne Selbstauflösung, sondern eine Integration, in welcher das Subjekt in seinem Verhältnis zum Anderen und Negativen zugleich mit sich eins wird. Im Naturrechtsaufsatz hat Hegel diese Figur als „Tragödie im Sittlichen“ beschrieben, worin die Versöhnung durch die Einsicht in die Notwendigkeit und das Recht des Anderen zustande kommt: Die Sittlichkeit, die dem Negativen und Fremden ,einen Teil ihrer selbst überläßt und opfert", kommt aus der Trennung zur Vereinigung mit sich selbst zurück. ${ }^{18}$ Es ist die allgemeine Figur der Entäußerung und Rückkehr, des Hinaustretens in die Objektivität und der Wiederaneignung, die Hegel hier zur letzten Negativität zuspitzt - worin „,das Absolute [...] sich ewig in die Objektivität gebiert, in dieser seiner Gestalt hiermit sich dem Leiden und dem Tode übergibt und sich aus seiner Asche in die Herrlichkeit erhebt." $" 19$

Mit unterschiedlicher Akzentuierung wird in Variationen dieses Modells das Andere in ein Ganzes aufgenommen und zwischen den antagonistischen Potenzen ein geregeltes Verhältnis etabliert. In der attischen Tragödie sieht Nietzsche die beiden Urtriebe des Dionysischen und des Apollinischen zu einem ,geheimnissvolle[n] Ehebündniss“ zusammenwirken, worin die Auflösungskraft nur in dem Maße tätig wird, wie sie von der Verklärungs- und Gestaltungsmacht wieder aufgenommen und zusammengehalten werden kann. ${ }^{20}$ Georg Simmel hat die Vergegenständlichung des Lebens, die in der kulturellen Produktion stattfindet, in den Horizont einer „Tragödie der Kultur“ gerückt und darin die Verselbständigung des Anderen betont, das nicht umstandslos in den Kreislauf des Lebens zurückgeführt werden kann. ${ }^{21}$ Dieser ,tragischen' Lektïre des Äußerungsmodells stehen andere Konzepte gegenüber, welche die Objektivation des Lebens als Medium des Selbstausdrucks und der Selbstverständigung zur Geltung bringen (Dilthey, Ricœur, Herder, Charles Taylor) und darin Simmels Sichtweise teils

17 Hegel, Phän., TWA 3, S. 36.

18 Hegel, NR, TWA 2, S. $494 \mathrm{f}$.

19 Hegel, NR, TWA 2, S. 495.

20 Nietzsche, GT 25, KSA 1, S. 42.

21 Vgl. Georg Simmel: Der Begriff und die Tragödie der Kultur. In: ders.: Das individuelle Gesetz. Philosophische Exkurse. Hg. v. Michael Landmann. Frankfurt a.M. 1968, S. 116-147. 
direkt widersprechen (Cassirer). ${ }^{22}$ Im Ganzen wird es zu einer offenen Frage, in welcher Weise solche Entzweiung zustande kommt und inwiefern das Leben die Trennung zu übergreifen, den Widerspruch zu versöhnen vermag. In äußerster Zuspitzung ist die Figur bei Hegel gezeichnet, wenn er die Konfrontation mit Schmerz und Tod mit dem Anspruch auf höchste Vereinigung verknüpft. Die kritische Rückfrage etwa bei Adorno - betrifft das Ernstnehmen der Differenz wie die postulierte Einheit gleichermaßen.

\subsection{Aufsichnehmen der Schuld und Läuterung}

Wie die Schuldhaftigkeit eine Vertiefung des erlittenen Negativen darstellt, geht das Aufsichnehmen der Schuld weiter als das Ertragen des zugefuigten Leids. Es beinhaltet ein in sich gebrochenes, sich gleichsam widersprechendes Zustimmen: Die Strafe ist nicht bloße Folge, sondern Beweisgrund der Schuld, welche der Held eingestehen und auf sich nehmen muss, wie Antigone aus der Tatsache, dass die Götter in die Bestrafung des gottesfürchtigen Tuns einwilligen, „duldend wohl gestehn" muss, dass sie fehlte. ${ }^{23}$ Zur schicksalhaften Unentrinnbarkeit gehört nicht nur das Eintreten von nicht-intendierten Folgen des Handelns, sondern dessen eigene Umdefinition, gegen die ursprüngliche Intention, in ein schuldhaftes Tun. Indem der Mensch die Verantwortung für die Tat auf sich nimmt, wird er selbst zum Urheber des ihm zugefügten, dadurch noch potenzierten Leidens: „von allem Leid am meisten schmerzt, was sich als selbstgewählt erweist." "24 Das Selbst-GewähltHaben des Nicht-Gewollten ist der Kern des tragischen Handelns, das darin nicht nur der Abgründigkeit der Schuld, sondern zugleich des Leidens begegnet. Dazu stehen zu können macht die fast übermenschliche Größe des tragischen Helden in seinem Eigensinn aus. Zugleich ist dieses Einstehen für die eigene Schuld und das eigene Leiden eine Weise der Bewältigung. Das Anerkennen der Notwendigkeit nicht nur als eines Äußeren, den Menschen Überwältigenden, sondern als eines aus ihm selbst Kommenden und seinem Wesen Zugehörigen, ist eine Weise der

22 Vgl. Ernst Cassirer: Die „Tragödie der Kultur“. In: ders.: Zur Logik der Kulturwissenschaften. Fünf Studien (Göteborg 1942). Darmstadt 1961, S. 103-127.

23 Sophokles, Antigone, V. 926.

24 Sophokles, König Oidipus, V. $1230 \mathrm{f}$. 
Befriedung, die im Maße der Versöhnung mit der Welt auch eine Versöhnung mit sich selbst bewirkt.

\subsection{Erkenntnis und Deutung}

„[N]icht das Leiden selbst war das Problem, sondern dass die Antwort fehlte für den Schrei der Frage, wozu leiden?"“, meint Nietzsche im Blick auf die Funktion der asketischen Ideale: „Die Sinnlosigkeit des Leidens" war das Problem, das durch eine Deutung bewältigt wurde, welche das Leiden auslegte und die Leere ausfüllte: „[D]er Mensch war damit gerettet, er hatte einen Sinn “. ${ }^{25}$ Wenn Leiden generell etwas ist, das dem Subjekt nicht nur widerfährt, sondern zu dem es sich immer auch verstehend-interpretierend verhält, so werden Erkenntnis und Deutung zum eigenen Moment, das die erlebte Negativität mit ausmacht und verschieden prägt - je nachdem ob solches Verstehen zur Bewältigung, zur versöhnenden Erkenntnis, zur resignierenden Akzeptanz, zur rationalisierenden Verdrängung oder im Gegenteil zur Verzweiflung angesichts des Nichtbewältigbaren führt. Solche Deutung des Leidens wird sowohl individuell geleistet wie in kulturellen Mustern tradiert, sie kristallisiert sich in Formen der künstlerischen Darstellung wie der theoretischen Reflexion. In einem gewissen Maße ist kulturelle Arbeit im Ganzen, sofern sie der Selbstverständigung des Menschen dient, auch mit Leiden befasst. Die Beschreibungen der Welt und des Menschen durchdringen sich mit unserem Verständnis des Negativen und Nichtseinsollenden. Gleichzeitig steht Negativität für jene Grenze, an welcher kulturelle Konstruktion und Nichtkonstruierbares aufeinander stoßen, wenn Leiden - etwa im physischen Schmerz - als ursprüngliches, nicht durch Deutung zu bewältigendes begegnet.

Neben der kulturellen Praxis im Allgemeinen sind es spezifische Formen der Reflexion, die mit der Frage nach dem Leiden befasst sind. Religionen und Philosophien sind Bemühungen, mit der Konfliktualität des Lebens und den Aporien widersprüchlicher Selbstbeschreibungen zurechtzukommen. Theodizeen sollen die Rechtfertigung Gottes angesichts des Übels in der Welt leisten und damit ein im Ganzen rationales Wirklichkeitsverständnis herstellen. In prägnanter Weise ist moderne Geschichtsphilosophie nach Kant und Hegel dem Anspruch verpflichtet, im Schicksal der Menschheit den Gang der Vernunft aufzuweisen und

25 Nietzsche, GM III, 28, KSA 5, S. 411. 
damit über Resignation und Trostlosigkeit hinwegzukommen; allgemein hat Hegel das Ziel der Philosophie darin gesehen, den Menschen mit der Welt zu versöhnen. In alledem kann die aporetische Erfahrung des Sinnlosen selbst zum Movens kultureller Sinnstiftung werden. ${ }^{26}$ Dieser Zusammenhang ist von U. Wolf als Leitidee einer Philosophie ausgearbeitet worden, welche sich durch die Frage nach dem guten Leben bestimmt, worin die Sinnfrage als Antwort auf existentielle Aporien fungiert. ${ }^{27} A$ fortiori ist ein Denken, das sich der Erfahrung des Absurden und Negativen aussetzt, auf diesen Weg gewiesen. Immer ist dabei die Frage gestellt, wieweit Erkenntnis und Deutung eine echte Bewältigung ermöglichen oder in Täuschung und Verdrängung münden. Unverkennbar gehört der Ausblick auf Versöhnung vor dem Hintergrund eines Wohlwollens der Götter (auch wenn deren Wille undurchschaubar bleibt) zum Ausgang klassischer Tragödien. ${ }^{28}$

\subsection{Selbstwerdung im Tragischen}

In all diesen Bestimmungen erweist sich das Tragische als Ort und Medium subjektiver Selbstwerdung. Es ist eine Selbstwerdung aus dem Negativen, die sowohl im Tun und Erleiden wie im Verstehen und Deuten stattfindet. Das Tragische liegt nicht nur in der objektiven Verfassung menschlicher Existenz, sondern beinhaltet ebenso die Art und Weise, wie Subjekte mit dieser handelnd, erkennend und deutend umgehen, wie sie Negativität erfahren, diese Erfahrung ausformulieren und in ihr Wissen und Verstehen integrieren. Erst über diese Reflexivität gewinnt Tragik ihre Pertinenz und Tiefe. Menschliches Selbstsein vollzieht sich über eine Hermeneutik des Selbst, die einen vielschichtigen

26 Jörn Rüsen bringt dies auf die Formel: „Leiden gebiert Sinn“ (Jörn Rüsen: Sinnverlust und Transzendenz - Kultur und Kulturwissenschaft am Anfang des 21. Jahrhunderts. In: Friedrich Jaeger/Jörn Rüsen (Hg.): Handbuch der Kulturwissenschaften. Bd. 3: Themen und Tendenzen. Stuttgart 2004, S. 533-544, hier S. 542); vgl. Emil Angehrn: Hermeneutik und Kritik. In: Rahel Jaeggi/Tilo Wesche (Hg.): Was ist Kritik? Frankfurt a.M. 2009, S. 319-338.

27 Vgl. Ursula Wolf: Die Philosophie und die Frage nach dem guten Leben. Reinbek 1999, S. 19-21, S. $123-139$ u. S. 152-154.

28 Zumal trifft dies zu für Sophokles und Aischylos, während bei Euripides (z. B. in Hippolytos und Die Bakchen) teils eine negativ-pessimistische Theodizee vorherrscht, worin die Menschen als bloßer Spielball der Willkür und Grausamkeit der Götter erscheinen. Vgl. hierzu in diesem Band den Beitrag von Bernhard Zimmermann: Über das Tragische bei den Griechen. 
Prozess der Selbsterkenntnis, Selbstinterpretation und Selbstaneignung umfasst.

Es ist eine Bewusstwerdung, in welcher Aporien reflektiert und Konflikte durchlebt werden und die nicht als einfache Introspektion zustandekommt, sondern als langwieriger, schmerzvoller Prozess der Einsicht und Erfahrung gegen inneren wie äußeren Widerstand realisiert werden muss. Hegel spricht geradezu von einer zweiten Hauptkollision des Tragischen - neben der ersten zwischen natürlicher Sittlichkeit und Staat -, die durch das „Recht des wachen Bewußtseins“"29 gegeben ist, welches sich nicht nur von der bewusstlosen Ausführung des Willens der Götter emanzipiert, sondern auch gegen Verhüllung und Verblendung durchsetzen muss. Der Topos von Blindheit und Sehen (exemplarisch im Ödipus-Drama) ist ein eindrucksvolles Zeugnis dieses Motivs. Inhaltlich hat die Bewusstwerdung den zweifachen Fokus einer Verständigung über den Menschen als solchen wie über die Identität des Einzelnen und sein individuelles Los. Ödipus’ Antwort „Es ist der Mensch!“ ist nur eine unzulängliche Überwindung der bedrängenden Unwissenheit, hohl und abstrakt gegenüber dem, was zu wissen nottut und was das Individuum mit sich selbst, in seiner Singularität, konfrontiert. ${ }^{30}$

Dass das Wissen Widerstand zu überwinden hat, ist in der Negativität begründet, die den Kern der gesuchten Selbsterkenntnis ausmacht. Wie der tragische Held im Leiden lernen und aus der Zerrissenheit zur Läuterung gelangen soll, so ist sein Verständnis des Menschen eines, das seine Wurzel in der Erfahrung des Negativen, des Nichtseinsollenden und Nichtgewollten, hat. Die Tragödie inszeniert einen Weg der Selbstwerdung im Modus einer negativistischen Erkenntnis seiner selbst in seiner Ohnmacht und Widersprüchlichkeit. Nur ein Wissen, das von dieser Negativität nicht absieht, kann Grundlage einer Selbstdeutung werden, in welcher der Mensch ein wirkliches Verständnis seiner selbst gewinnt. Nur auf diesem Weg kann Verdrängtes zurückgewonnen, Ausgeschlossenes wiederaufgenommen werden. Außerhalb der klassischen Tragödie schließt sich die Frage an, in welcher Dimension solche Negativität in den Blick kommt - im Negativen der realen Welt, in der konstitutiven Endlichkeit des Daseins oder in der von Religion und Metaphysik reflektierten, die Grenze des philosophischen Begriffs be-

29 Hegel, Ästh. III, TWA 15, S. 545.

30 Vgl. Klaus Heinrich: arbeiten mit ödipus. Begriff der Verdrängung in der Religionswissenschaft (Dahlemer Vorlesungen 3). Hg. v. Hans-Albrecht Kücken u. a. Basel/Frankfurt a.M. 1993, S. 112-114 u. S. 134-136. 
rührenden Faktizität des Leidens und des Bösen. Die Frage verbindet sich mit jener nach der Reichweite und den Grenzen des Tragischen selbst.

\section{Grenzen des Tragischen}

\subsection{Immanente Vollendung des Tragischen - Läuterung und Versöhnung}

Es mag zum Teil eine Frage der Terminologie sein, ob man die zuletzt sichtbar gewordene Umkehrung als eigene Vollendung des Tragischen oder als dessen Überwindung bezeichnet. Im Blick auf klassische Tragödien wie Ödipus auf Kolonos oder die Eumeniden liegt es nahe, in der Wiedervereinigung und Versöhnung ein immanentes Telos, ein $\mathrm{Zu}$ Ende-Führen des Tragischen zu sehen. Dem entspricht nicht zuletzt die Bestimmung, die Aristoteles von der Wirkung der Tragödie gibt. Durch „Jammer und Schaudern“ bewirkt sie „eine Reinigung" von der Angst und vom Schrecken, welche die tragische Verwicklung hervorruft. ${ }^{31}$ Die Tragödie als kultische Veranstaltung erfüllt für die Gemeinschaft eine bestimmte Funktion, zu der sowohl die Erschütterung wie deren Überwindung, die Beruhigung und Versöhnung gehören. Im Hindurchgehen durch den Schrecken und das Mitleiden soll die Seele geläutert werden, im Aushalten von Angst soll sich der Mensch von Ängsten befreien. Dieser Ausblick geht über die innere Sühne des Helden hinaus. Er meint in umfassendem Sinn eine Wiederherstellung der Einheit auf höherer Ebene, wie sie exemplarisch in Sophokles' letztem Werk, Ödipus auf Kolonos, begegnet: als Versöhnung des Helden mit sich, mit seinem Schicksal, mit der Welt und den Göttern, schließlich als kosmische Versöhnung zwischen irdischen und himmlischen Mächten und, nach Ödipus' Sterben, als Versöhnung mit dem Tod und Verzicht auf Klage und Trauer. ${ }^{32}$ Es ist eine Versöhnung, die nichts von der Tiefe der Schuld, der Härte des Leidens und der Zerrissenheit rückgängig macht und doch im Ganzen eine Lösung, eine Befriedung bewirkt. In diesem $\mathrm{Zu}$-EndeFühren des Tragischen kann man die Vollendung der Tragödie sehen, die zugleich ihr Abschluss und ihr Ende ist.

Von dieser immanenten Vollendung sind jene Formen des Umgangs mit dem Tragischen zu unterscheiden, die gleichsam von außen, in je

31 Aristoteles, Poetik, Kap. 6, $1449 \mathrm{~b} 27$.

32 So die abschließende Ermahnung des Theseus an Ödipus' Töchter (Sophokles, Oidipus auf Kolonos, V. 1751-1753). 
anderer Weise, zu dessen Negativität Stellung nehmen: als dessen Überwindung und Aufhebung, als seine Auflösung, als Restitution des Negativen jenseits tragischer Vermittlung und Versöhnung.

\section{2 Überwindung und Aufhebung des Tragischen}

Die erste Linie, auf der sich gleichsam das Potential des Tragischen in affirmativer Form weiter- und höherentwickelt, ist durch Hegels $\mathrm{Ne}$ gativitätstheorie angedeutet. In der spekulativen Dialektik ist die Tragik aufgehoben - überwunden und in ein Höheres aufgenommen. Diese Aufhebung lässt sich in systematischer wie historischer Perspektive lesen. Systematisch geht sie mit dem Übergang von der Individualität zur Subjektivität einher, zu jener Form des Selbstverhältnisses, das in der Lage ist, die Beziehung zum Anderen und zum Negativen innerhalb seiner zu enthalten und zu übergreifen. Sie verbindet sich mit dem Ende des Mythos und dem Wissen vom notwendigen Untergang der Individualität, wie es Hegel als Melancholie der Schönheit im stillen Zug der Trauer an den griechischen Götterbildern wahrzunehmen meint. ${ }^{33}$ Die Aufforderung an die Philosophie, den ,unendlichen Schmerz" als „Moment der höchsten Idee" zu denken, ist von der Überzeugung getragen, dass erst aus dem absoluten Leiden ,die höchste Totalität in ihrem ganzen Ernst und aus ihrem tiefsten Grunde [...] in die heiterste Freiheit ihrer Gestalt auferstehen kann und muß." Der ,spekulative Karfreitag" weist auf den Konvergenzpunkt von christlicher Religion und moderner Philosophie, der zugleich deren gemeinsame Distanz vom tragischen Bewusstsein markiert. ${ }^{34}$ Die Versöhnung, welche die Erlösung gewährt und welche die spekulative Philosophie zu fassen sucht, ist von anderer Tiefe als die Läuterung, zu der die immanente Bewältigung des Schmerzes im Tragischen führt. Die Moderne ist jenseits des Tragischen, sofern dessen radikale Entzweiung nur ein Vorletztes, nicht das abschließende Ganze ist und die im Geiste erreichte Vereinigung die tragische Versöhnung transzendiert.

33 Vgl. Hegel, Ästh. II, TWA 14, S. 85 f. u. S. 104. - Ähnlich stellt Schelling den "Zug tiefer Schwermuth, der durch das ganze Heidenthum geht" in der Ahnung vom Untergang der Götter heraus (Schelling, SW XII, S. 346).

34 Hegel, GuW, TWA 2, S. 287-433, hier S. 432 f. 


\subsection{Auflösung des Tragischen}

Der andere Ausgang des Tragischen ist seine Auflösung und Unterlaufung. Sie tritt in dem Maße ein, wie die Konfliktualität und Entzweiung ihre innere Notwendigkeit, der Wille seine Selbstwidersprüchlichkeit verliert. Wenn Hegel das Schicksal des Sokrates als „echt tragisch“ bezeichnet, weil es aus der Notwendigkeit des höheren Prinzips und dem damit verbundenen Konflikt berechtigter Ansprüche resultiert - „tragisch nicht im oberflächlichen Sinn des Worts, wie man jedes Unglïck - wenn jemand stirbt, einer hingerichtet wird - tragisch nennt; dies ist traurig, aber nicht tragisch ${ }^{\text {“35 }}$-, so wird der immanente Widerspruch zum entscheidenden Merkmal. Schon in der Transformation der alten Tragödie, wenn ,,bei Euripides die Götter sozusagen nach oben, in völlige Unbegreiflichkeit, die Heroen nach unten, in allzu krasse Menschlichkeit“" auseinandergehen, ${ }^{36}$ wird die Einheit, welche der Antithetik vorausliegt, gelockert. In vielen Varianten kann die Wendung ins Kontingente und Unverbindliche, die Abschwächung der Konfliktualität zum Oberflächenphänomen, die Überführung ins Ästhetische durchgespielt werden, wodurch die Widersprüchlichkeit des menschlichen Selbstseins ihren Stachel, die tragische Konstellation ihre Härte verliert. Einen Aspekt dieses Wandels repräsentiert der Übergang von der Tragödie zur Komödie. Im Ganzen geht es um einen Prozess, der mit der Aushöhlung des substantiellen Grundes auch dessen Zerrissenheit suspendiert. In gesteigerter Form haben Konzepte der Postmoderne in der Bejahung von Differenz und Pluralität, im Lob des Zufalls und der Oberfläche diese Gegenwendung zum Ausdruck gebracht, in welcher die Differenz ihr konfliktives Potential, das Negative seine aporetische Intransigenz verliert.

35 Hegel, PhGesch I, TWA 18, S. 514 u. S. 446. - Zum Verhältnis von Trauer und Tragik in der neueren Dichtung und Philosophie vgl. Marc Sagnol: Tragique et tristesse. Walter Benjamin archéologue de la modernité. Paris 2003; ders.: Tragik und Trauer bei Benjamin. In: Kathy Zarnegin (Hg.): Buchstäblich traurig. Basel 2004, S. 55-85.

36 Fritz Graf: Griechische Mythologie. Eine Einführung. München/Zürich 1985, S. 167. 


\section{Negativismus und Tragik}

Schließlich ist eine dritte Denkform jenseits des Tragischen zu nennen, die sich von dessen Aufhebung in ein Höheres wie von seiner Suspendierung und Unterlaufung gleichermaßen abhebt. Es geht um ein Denken, das die im Tragischen erfahrene Negativität ernst nimmt, ohne sie in eine - sei es der Tragik immanente, sei es sie transzendierende Versöhnung zu überführen. Zur Diskussion steht ein ,negativistisches Denken, ${ }^{37}$ das sich über den Menschen und die Wirklichkeit im Ausgang von der Konfrontation mit dem Negativen verständigt ${ }^{38}$ und darin wesentliche Motive des tragischen Denkens teilt. Von der Tragödie wie von der Erlösungsreligion und der spekulativen Dialektik unterscheidet es sich dadurch, dass es weder von der Gewisheit des letztlichen Versöhntseins der Welt - wie die Religion und Hegels System - noch vom Vertrauen in die zuletzt herrschende Gerechtigkeit des Schicksals und der Götter-wie die Tragödie bei Aischylos und Sophokles - getragen ist. Es setzt sich der Erfahrung eines Negativen aus, das als nicht-versöhntes das irreduzibel Andere des rationalen Begriffs bleibt und - so Adorno - „dem spekulativen metaphysischen Gedanken die Basis seiner Vereinbarkeit mit der Erfahrung" entzieht. ${ }^{39}$ Es geht um die Konfrontation mit einem Negativen, das nicht in ein umfassenderes Ganzes aufgehoben oder - so Marx' Vorwurf an Hegel - auf eine tiefere Einheit im Wesen zurückgeführt werden kann, ${ }^{40}$ sondern fern jeder diskursiven und praktischen Bewältigung allein Gegenstand der Abwehr, der ,unbeirrten Negation“41 ist. Gleichwohl gehört es zu den Herausforderungen an eine Hermeneutik des Selbst, auch dieses Negative in den Kreis dessen einzubeziehen, wovon das Subjekt Rechenschaft ablegen muss, wenn es sich über sich selbst und über die Welt verständigen will. Das Selbstverhältnis des Subjekts ist nicht nur mit dessen konstitutivem Bezug zum Anderen,

37 Zum Begriff vgl. Michael Theunissen: Negativität bei Adorno. In: Ludwig von Friedeburg/Jürgen Habermas (Hg.): Adorno-Konferenz 1983. Frankfurt a.M. 1983, S. $41-65$.

38 Vgl. Emil Angehrn: Negativistische Hermeneutik. Zur Dialektik von Sinn und Nicht-Sinn. In: Andreas Hetzel (Hg.): Negativität und Unbestimmtheit. Beiträge zu einer Philosophie des Nichtwissens Festschrift für Gerhard Gamm. Bielefeld 2009, S. 21-40.

39 Theodor W. Adorno: Negative Dialektik. Frankfurt a.M. 1975, S. 354.

40 Karl Marx: Kritik des Hegelschen Staatsrechts (1843). In: Karl Marx/Friedrich Engels: Werke. Berlin 1957 ff., Bd. 1, S. 203-333, hier S. 290 u. S. 295 f.

41 Adorno: Negative Dialektik, S. 162. 
sondern mit jener Vertiefung der Alterität zusammenzudenken, für welche die Negativität steht. Das beschädigte Leben, Krankheit, Leiden und Tod müssen in das Verständnis des Selbst aufgenommen werden. Dabei ist zu differenzieren, welchen Status das Negative hat, mit dem wir in solchem Verstehen konfrontiert sind. Teils ist es die konstitutive Negativität der Existenz, die Endlichkeit und der Mangel, die menschlichem Leben unabdingbar sind, teils das Negative der bestehenden Welt, das als Entfremdung, Unrecht, Gewalt und Destruktion erfahren wird, teils das ,metaphysische' Negative: die unerlöste Natur, das Böse, die Sinnlosigkeit der Existenz und des Alls. Es hängt gewissermaßen von einer fundamentalphilosophischen Option ab, in welcher Tiefe und Durchgängigkeit subjektives Selbstsein dem Negativen ausgesetzt ist und sich in der Auseinandersetzung mit dem Negativen behauptet.

In radikalster Form tritt uns dieses in Gestalt des Bösen und des Leidens entgegen - Herausforderungen für das praktische Leben wie für die Religion und den philosophischen Begriff. P. Ricœur hat diese Herausforderung in seiner Schrift Finitude et culpabilité (1960) so formuliert, dass die philosophische Reflexion über den Menschen nur in der Lage ist, die Fehlbarkeit als Wesensmerkmal der menschlichen Existenz, d.h. die Möglichkeit, aber nicht das Faktum der Schuld und das kontingente Ereignis des Bösen zu denken. ${ }^{42}$ Über dieses verständigt sie sich vielmehr auf dem Umweg über die kulturellen Dokumente der Menschheit, in der Lektüre der Erzählungen, Mythen und Symbole, in denen die Völker Zeugnis ablegen von ihrer Erfahrung von Schuld und Leid. Es ist eine Erfahrung, die sich der rationalen Bewältigung entzieht und von der sie nur unzulänglich Rechenschaft ablegen können. In gewisser Weise stellt das Übel, das die Menschen nicht verschulden, sondern das sie erleiden - das ,Leiden', nicht das ,Böse“ -, die noch radikalere Herausforderung für das Denken dar. Das unschuldige Leiden ist der Skandal der Vernunft und Inbegriff des Sinnlosen - nach E. Lévinas das Nichtassimilierbare und Unannehmbare - ,l'inassumable“ -, das sich nicht nur faktisch dem rationalen Diskurs entzieht, sondern sich ihm antithetisch entgegensetzt. Es ist nicht nur ohne Rechtfertigung und Sinn, das Sinn-lose, sondern steht gewissermaßen für die aktive Bestreitung, ,Verneinung und Verweigerung des Sinns“ ${ }^{43}$ die jeder logi-

42 Vgl. Paul Ricœur: Philosophie de la volonté. Tome I: Le volontaire et l'involontaire. Paris 1950; Tome II: Finitude et culpabilité. Paris 1960.

43 Emmanuel Lévinas: La souffrance inutile. In: ders.: Entre nous. Essais sur le penser-à-l'autre. Paris 1991, S. 107-120, hier S. 107 [Übers. v. Verf.]. Vgl. Paul 
schen Negation vorausliegt. ${ }^{44}$ Hier scheinen wir auf eine äußerste Grenze, eine letzte Negativität zu stoßen, die in keiner Weise transzendiert oder vom Denken in eine höhere Synthesis aufgehoben werden kann.

Abstrakt können wir den Ausgang vom Negativen unter zwei Perspektiven, einer internen und einer externen, thematisieren. Auf der einen Seite haben wir die in der Existenzphilosophie reflektierte Erfahrung der Nichtigkeit - der Haltlosigkeit, Angst, Verzweiflung, Sinnlosigkeit -, in welcher das Subjekt mit dem eigenen Sein, der ontologischen Verfassung seiner Existenz zu tun hat. Das Andere ist das ihm von außen entgegentretende Negative, dem es ausgesetzt ist - Gewalt, Vernichtung, Leiden, das Böse. Traumatische Erlebnisse verkörpern diese Erfahrung in extremer Gestalt, exemplarisch auch darin, dass sie typischerweise nicht nur jede rationale Bewältigung unterlaufen, sondern radikaler sich der Erinnerung, der Erzählung, der Repräsentation als solcher verweigern. In der Sprach- und Bildlosigkeit scheint das Nichtverstehenkönnen absolut.

Darin zeigen sich Nähe und Ferne zum Tragischen. Die Nähe liegt im ungeschützten Exponiertsein, im Ernstnehmen der radikalen Infragestellung, in der Begegnung mit Unrecht und Tod. Wie in der Tragik geht es dem Negativismus um eine Konfrontation mit dem Negativen, die sich nicht kontingenterweise einstellt, sondern die gleichsam aus einer inneren Unstimmigkeit des Wirklichen, einer Widersprüchlichkeit des menschlichen Seins und Wollens erwächst und die gleichwohl den Menschen in seinem Wesen betrifft. Die Distanz zum Tragischen liegt in der Verschärfung des aporetischen Umgangs mit solcher Negativität: darin, dass es sich um ein Negatives handelt, das nicht im tragischen Leiden irgendwie pazifiziert, eingedämmt werden kann. Es geht um ein unversöhntes und nicht-versöhnbares Leiden, um eine nicht-dialektisierbare Negativität, die nicht innerhalb einer im Ganzen herrschenden Rechtsordnung, eines göttlichen Kosmos ertragen und aufgenommen, zum Medium eines Höheren geläutert werden kann. Wie das Negative einerseits, als existentielle Nichtigkeit, der spezifischen Konflikt- und Schuldhaftigkeit des Tragischen vorausliegt, so weist es andererseits, in der Abgründigkeit des Bösen und des Leidens, über die tragische Konstellation hinaus. Die Vertiefung des Negativen radikalisiert die Aporetik

Ricœur: Le Mal, un défi à la philosophie et à la théologie. Genf 1986; ders.: Le scandale du mal, in: Esprit (Juli/August 1988), S. 59-63.

44 Vgl. Lévinas: La souffrance inutile, S. 108: „Cette négativité du mal est, probablement, source ou noyau de toute négation apophantique." 
der Deutung, die ohne integrierenden Sinnhorizont und ohne abschließende Einwilligung bleibt.

Indessen ist das Negative trotz der Nichtintegrierbarkeit nicht das berïhrungslos Andere. Das im Extrem nicht-repräsentierbare, ,namenlose“ Leiden bleibt eines, das dennoch das Subjekt in seinem Innersten betrifft, das sein Verstehenkönnen und sein Verstehenmüssen herausfordert. Für Lévinas ist es der Perspektivenwechsel von der theoretischen zu einer zwischenmenschlich-ethischen Haltung, der dem Leiden seine Sinndimension zurückgibt. ${ }^{45}$ Doch kann man sich fragen, ob eine solche Restitution nicht auch unabhängig vom Übergang zu einer Ethik der Verantwortlichkeit, innerhalb der Hermeneutik des Selbst als solcher, möglich ist. Wenn zu dieser Hermeneutik nach Ricœur die Dialektik zwischen dem Selbstverständnis und dem Bezug zum Anderen gehört, so scheint es richtig, diesen Bezug auf jene letzte Alterität hin auszuweiten, die im Negativen als solchem liegt. Auch wenn sich dieses dem Bemühen widersetzt, es rational zu durchdringen und in ein affirmatives Ganzes aufzunehmen, sondern es im strikten Sinne fremd, nicht-assimilierbar bleibt, so ist es doch eines, dessen das Selbst gedenken, von dem es Rechenschaft ablegen muss, wenn es sich über sich und die Welt Klarheit verschaffen will. In seiner Selbstbesinnung kann das Subjekt weder von dem Übel absehen, dessen es schuldig ist, noch von jenem, das es erleidet: $\mathrm{Zu}$ seinem Selbst gehört das Gedächtnis dessen, was es weder wollen und ertragen noch verstehen oder rational bewältigen kann. Eine negativistische Hermeneutik, deren Kern das Verstehen seiner selbst im Angesicht des Negativen bildet, ist angestoßen durch Motive, die sie mit der Tragik teilt, und entfaltet sich in einem Raum, der sie zugleich über das tragische Denken hinausführt. 
